\title{
Control Design for the Interactive 3D-Pendulum Presented at the World Exhibition EXPO 2010*
}

\author{
Robert SEIFRIED**, Thomas GORIUS ${ }^{* *}$ and Peter EBERHARD** \\ ${ }^{* *}$ Institute of Engineering and Computational Mechanics \\ University of Stuttgart, Pfaffenwaldring 9, 70569 Stuttgart, Germany \\ E-mail: robert.seifried@itm.uni-stuttgart.de
}

\begin{abstract}
The German Pavilion on the World Exhibition EXPO 2010 in Shanghai showed an interactive 3D-pendulum as one of its main attractions. The pendulum consists of a sphere with $3 m$ diameter which is equipped with several hundred thousand LEDs and used as a projection screen. The sphere is mounted via a long bar at an electrically driven crosstable which is used for the excitation of the pendulum. The crosstable is located in a theater-like building where the visitors can follow the show. Thereby, the sphere performs large circular and pendular motions, while the crosstable can only perform very small motions. Also during some parts of the show the sphere should move in a certain way which is obtained by interpreting the visitors' action as a physical excitation. In this paper the system and control design are discussed and experimental results are presented.
\end{abstract}

Key words : Control, 3D-Pendulum, Swinging Motion, Tracking, Stabilization, Experiments

\section{Introduction}

During the World Exhibition EXPO 2010 in Shanghai the German Pavilion featured as its main attraction a large interactive 3D-pendulum. The pendulum consists of a sphere with $3 m$ diameter which is equipped with approximately 400000 LEDs. These are used as a 360 degree projection screen for a multimedia show. The sphere is mounted via a long thin bar and a Cardan joint with an electrically driven crosstable which is used for the excitation of the pendulum. The pendulum's length is $5.6 \mathrm{~m}$ and due to the large amount of LEDs and electronics the pendulum weights approximately $1.2 t$. The crosstable is integrated in the roof of a theater-like building where several hundred visitors can follow the show. Thereby, the sphere should perform large circular and pendular motion, while the crosstable should only perform very small motions. In such a way the illusion of a free moving sphere is achieved. Also, during some parts of the show the sphere should move in a certain direction which is obtained by interpreting the visitors' action as an interactive physical excitation.

The practical and widely used control-counterpart of this pendulum is a gantry crane, which is often used as example in control theory literature [1-6]. Despite the apparent similarities of these systems, these approaches from literature cannot be used for this pendulum. Roughly speaking, this is due to the directly opposite control objectives of gantry cranes and the EXPO-pendulum. In order to provide an impressive show the sphere should perform large displacements while ensuring only a very small motion of the crosstable.

In this paper the mechanical design and the control approach for the EXPO-pendulum are presented. This control, which is implemented on the real machine, is designed in such a way that it can be executed on certified industrial hardware in order to meet high safety and reliability requirements. Due to this use of industrial hardware the control input is the position of the crosstable and not the motor torque as it is often used in lab experiments. The control must assure fast start-up and breaking, while hard input constraints must be met.

${ }^{*}$ Received 22 Feb., 2011 (No. 11-0140) [DOI: 10.1299/jsdd.5.937]

Copyright $\subset 2011$ by JSME 
Based on a sophisticated control approach stabilization and tracking controllers are designed. With this control strategy it is not only possible to perform circular motion, pendular motion in arbitrary planes and quick breaking, but in addition during the motion it is possible to rotate the pendular plane, change from pendular motion to circular motion and vice-versa. Movies of the pendulum in action can be accessed via the website [7].

The paper is organized in the following way. First the detailed description of the mechanical system is given in Section 2. Then, in Section 3 the control approach for stabilization, pendular motion and tracking of circular motion of the pendulum, are presented. The efficiency, high accuracy and robustness of this control approach are demonstrated by experiments on the real pendulum, which are presented in Section 4. A short summary in Section 5 concludes this paper.

\section{System Description}

In order to highlight the difficulties and challenges in control design for this system, first, the mechanical design and the different control objectives are discussed.

\subsection{Mechanical Design}

Figure 1 is a picture of the EXPO pendulum in the German Pavilion in Shanghai, Fig. 2 shows close-ups of the former test rig in Stuttgart and in Fig. 3 a schematic representation of the EXPO-pendulum is given. The main parts of the system are the sphere, the connecting rod, a Cardan joint and the electrically driven cross table. The sphere (radius $r$, mass $m_{p}$, inertia tensor $\boldsymbol{I}_{p}$ ) is connected to a rod (mass $m_{s}$, inertia tensor $\boldsymbol{I}_{s}$ ) which is universal-mounted by a Cardan joint to the crosstable. The pendulum length, i.e. the distance from the Cardan joint to the sphere's center of mass, is denoted by $L$. The electrically driven crosstable consists of two carts (mass $m_{c}$ and $m_{r}$ ). In the pavilion the crosstable is integrated in the roof of a theater-like building where then several hundred visitors can follow the show. On the test rig the crosstable is mounted on a rigid steel structure. The system parameters are summarized in Table 1. It should be noted, that the given mass and inertia data are upper limits which were used in the design stage. Since the real values are only roughly known, the designed controller must be robust against uncertainties in these values. Since approximately 400000 LEDs are mounted on the surface of the sphere with $3 m$ diameter, the sphere has a very large inertia. In order to minimize the torsion on the rod during circular motion, a revolute joint is introduced between the sphere and the rod, allowing motion around the rod's axis. Due to this decoupling a slender rod of $8 \mathrm{~cm}$ diameter could be used. In this rod a power and data cable is integrated for the supply of the LEDs. Mainly caused by the big pendulum length $L=5.6 \mathrm{~m}$ the system's dynamical behavior is quite slow. In the case of a fixed mounting point the pendulum's eigenfrequency is approximately $0.21 \mathrm{~Hz}$.

A full simulation model of the pendulum is derived using the symbolic multibody system software Neweul- $\mathrm{M}^{2}$ [8]. The dynamical behavior is described by five degrees of freedom, the position of the mounting point $((x, y)$ in $(X, Y)$-coordinate axis), the orientation of the rod with the two Cardan angles $\alpha$ (around the $\mathrm{X}$-axis) and $\beta$ (around the direction $(0, \cos \alpha, \sin \alpha)^{T}$ in the $X Y Z$-coordinate system) and the rotation $\zeta$ of the sphere around the rod's axis. In the rod-fixed and sphere fixed coordinate system, respectively, the inertia tensors $\boldsymbol{I}_{p}, \boldsymbol{I}_{s} \in \mathbb{R}^{3 \times 3}$ with respect to the body's center of mass are

$$
I_{s}=\operatorname{diag}\left(I_{s x}, I_{s x}, I_{s z}\right), \quad I_{p}=\operatorname{diag}\left(I_{p}, I_{p}, I_{p}\right) .
$$

The acting forces on the carts of the crosstable are $F_{x}$ and $F_{y}$ in $X$ and $Y$-axis direction, respectively. These are the driving forces produced by the electric drives. Then, the nonlinear equations of motion are given as

$$
\boldsymbol{M}(\boldsymbol{q}) \ddot{\boldsymbol{q}}+\boldsymbol{k}(\boldsymbol{q}, \dot{\boldsymbol{q}})=\boldsymbol{B} \boldsymbol{u}
$$

with the vector of generalized coordinates $\boldsymbol{q}=\left[\begin{array}{ccccc}x & y & \alpha & \beta & \zeta\end{array}\right]^{T} \in \mathbb{R}^{5}$, the input vector $\boldsymbol{u}=\left[\begin{array}{lll}F_{x} & F_{y}\end{array}\right]^{T} \in \mathbb{R}^{2}$, the symmetric positive definite mass matrix $\boldsymbol{M}(\boldsymbol{q}) \in \mathbb{R}^{5 \times 5}$, the vector 


\section{Dynamios}

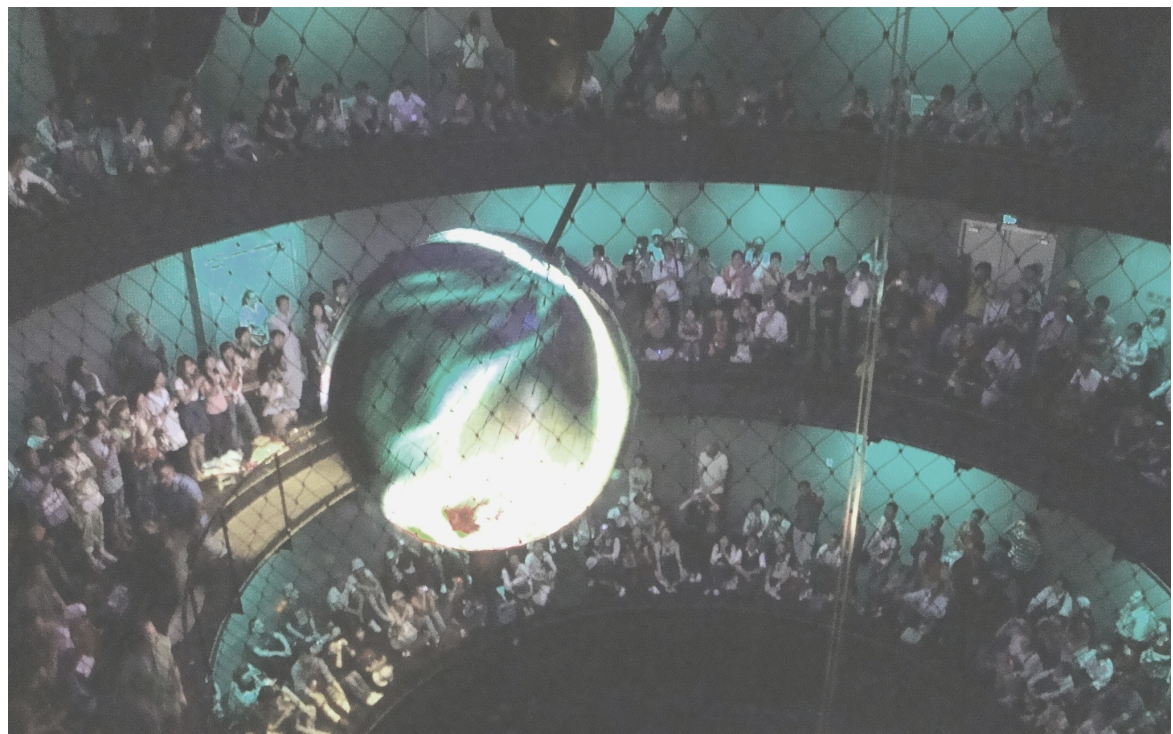

Fig. 1 The EXPO-pendulum in the German Pavilion, Shanghai

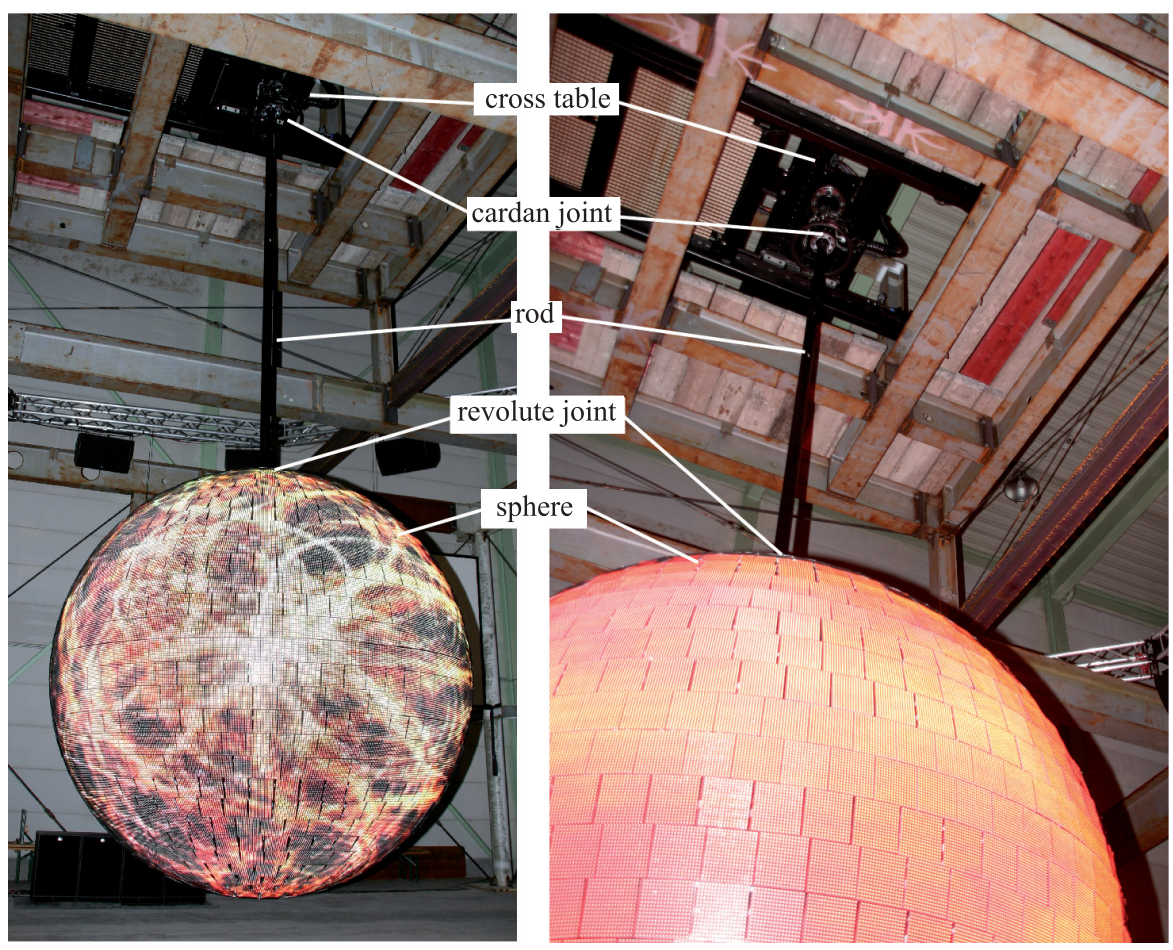

Fig. 2 Close-up of the EXPO-pendulum 


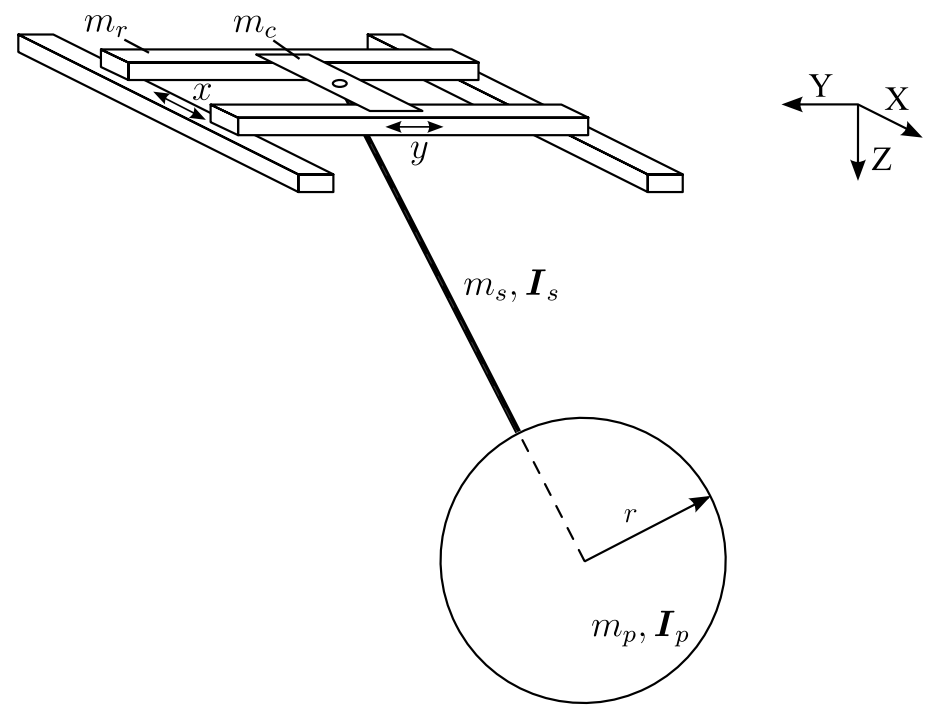

Fig. 3 Schematic representation of the EXPO-pendulum

Table 1 System parameters

\begin{tabular}{cc}
\hline parameter & value \\
\hline$m_{r}$ & $600 \mathrm{~kg}$ \\
$m_{c}$ & $380 \mathrm{~kg}$ \\
$m_{p}$ & $1200 \mathrm{~kg}$ \\
$m_{s}$ & $160 \mathrm{~kg}$ \\
\hline$I_{p}$ & $3000 \mathrm{~kg} \mathrm{~m} m^{2}$ \\
$I_{s x}$ & $224 \mathrm{~kg} \mathrm{~m} m^{2}$ \\
$I_{s z}$ & $0.16 \mathrm{~kg} \mathrm{~m} m^{2}$ \\
\hline
\end{tabular}

\begin{tabular}{cc}
\hline parameter & value \\
\hline$L$ & $5.6 \mathrm{~m}$ \\
$r$ & $1.5 \mathrm{~m}$ \\
$g$ & $9.81 \frac{\mathrm{m}}{\mathrm{s}^{2}}$ \\
\hline
\end{tabular}

of generalized forces $\boldsymbol{k}(\boldsymbol{q}, \dot{\boldsymbol{q}}) \in \mathbb{R}^{5}$ and the input matrix $\boldsymbol{B} \in \mathbb{R}^{5 \times 2}$. Since the EXPO pendulum has less control inputs than degrees of freedom it is a typical under actuated multibody system.

An analysis of the system shows, that the $\zeta$ degree of freedom is not controllable, representing a free rotation of the sphere around its vertical axis. Due to light friction in the joint and sliding contact of the power and data transmission this degree of freedom is damped. This rotation does not disturb the show since projections on the sphere can be shifted by this rotation, yielding the impression of a standing picture. Therefore, this degree of freedom is neglected in control design yielding the well-known pendulum-cart-system used to model overhead cranes. Then, the system features only 4 degrees of freedom with the generalized coordinates $\boldsymbol{q}=\left[\begin{array}{llll}x & y & \alpha & \beta\end{array}\right]^{T} \in \mathbb{R}^{4}$. The complete equation of motion of this reduced system is given in [9]. For the following control design and implementation the position of the sphere's center point described in the reference $X Y Z$-coordinate system is important and is described by

$$
\boldsymbol{w}=\left[\begin{array}{c}
w_{x} \\
w_{y} \\
w_{z}
\end{array}\right]=\left[\begin{array}{c}
x+L \sin \beta \\
y-L \sin \alpha \cos \beta \\
L \cos \alpha \cos \beta
\end{array}\right] .
$$

During operation of the pendulum several hard constraints must be fulfilled at all times. The maximal displacement of the crosstable is constrained by its design, mostly due to the request to achieve the illusion of a free moving sphere. Also due to the mechanical design of the crosstable with ball bearing spindles its velocity is limited. In addition the acceleration of the crosstable is limited in order to limit the maximal loads in the joint and bodies of the pendulum. Simulations show, that these are strongly influenced by the crosstable's acceleration. Also the drive torque, and thus the acting forces on the crosstable are limited. Finally, due to the design of the Cardan joint the Cardan angles are also limited. Thus, the constraints are 
given by

$$
\begin{aligned}
|x|,|y| \leq 0.35 m, \quad|\dot{x}|,|\dot{y}| \leq 0.4 \frac{m}{s}, \quad|\ddot{x}|,|\ddot{y}| \leq 0.5 \frac{m}{s^{2}}, \\
|\alpha|,|\beta| \leq 27^{\circ}, \quad\left|F_{x}\right|,\left|F_{y}\right| \leq 10 k N .
\end{aligned}
$$

It should be noted, that the mechanical and control system is designed to achieve even larger Cardan angles. However due the safety concept, large safety margins and end switches, the above stated limits must be met.

\subsection{Control Objectives}

In the pavilion the pendulum is placed in the center of a theater-like building with several hundred visitors following the show. During a several minute long show the sphere should perform large circular and pendular motions, while the crosstable should only perform very small motions. In such a way the illusion of a free moving sphere is achieved. Thus from a control point of view the main control objectives for the EXPO-pendulum are the following:

- $\quad$ stabilizing the equilibrium $(0,0,0,0)^{T}$,

- tracking a circular motion in the sense of making the sphere's center point $\left(w_{x}, w_{y}\right)$ to track circles in the $X Y$-plane with slowly variable radius, and

- achieving a pendular motion of the sphere's center point $\left(w_{x}, w_{y}\right)$ in the along the $Z$-axis rotated $X Z$-plane with variable amplitude.

Note, that stabilization of the system mainly means to bring the pendulum from an arbitrary motion into rest. Due to the limit on the Cardan angles the maximum radius and amplitude of the sphere's center is approximately $2.55 \mathrm{~m}$. During operation of the pendulum the desired motion is demanded online by a master system, which controls the complete multimedia show. Thus the controller must be able to switch between these kind of motions at any time. Of course the transient behavior of the closed loop should be as fast as possible because time is a leading factor during this show. This is especially important for the interactive part of the show. Thereby, the commands for the desired pendulum motion are obtained by interpreting the visitors action as control input, yielding an interactive experience. For example, the plane in which the swinging motion occurs can be rotated in the direction of the highest noise level which is produced by the visitors.

\section{Controller Design}

The EXPO-pendulum is a nonlinear system. A nonlinear controller is proposed in [9], where also its efficiency is demonstrated by simulation. However, a linear controller is presented in this paper, since the nonlinear controller cannot be implemented on the real system. This is due to the fact that the EXPO-pendulum is a large device where a lot of safety and reliability guidelines must be meet. Therefore, the whole system is only allowed to contain certificated parts, e.g. electronic devices, safety systems, etc. Commercial components like electric drive trains as used for common industrial applications can be combined well with such certificated devices which makes it easier to design a safety control and communication. The practical problem is that the drive train used for the EXPO-pendulum cannot be controlled by demanding a certain drive torque, which is equivalent to an acting force on the cart, because of severe communication and safety control problems. Therefore, the internal position and velocity cascade control of the drives must be used. Hence, the displacements of the carts $x$ and $y$ must be considered as the input of the dynamical system instead of the acting forces on the carts. The use of the internal position controller has the main advantage that the state constraints (4) are now input constraints which can be incorporated much easier. In addition, model uncertainties of the carts' dynamical behavior such as friction or elasticity of the drive train are directly compensated by this cascade control. For the control design a multibody system with generalized coordinates $\boldsymbol{q}=\left[\begin{array}{cc}\alpha & \beta\end{array}\right]^{T} \in \mathbb{R}^{2}$ and the cart position corresponding 
to rheonomic constraints is derived which yields

$$
\left[\begin{array}{cc}
m_{33} & 0 \\
0 & m_{44}
\end{array}\right]\left[\begin{array}{l}
\ddot{\alpha} \\
\ddot{\beta}
\end{array}\right]+\left[\begin{array}{c}
k_{3}(\alpha, \dot{\alpha}, \beta, \dot{\beta}) \\
k_{4}(\alpha, \dot{\alpha}, \beta, \dot{\beta})
\end{array}\right]=-\left[\begin{array}{cc}
0 & m_{23} \\
m_{14} & m_{24}
\end{array}\right]\left[\begin{array}{c}
\ddot{x} \\
\ddot{y}
\end{array}\right]
$$

where

$$
\begin{aligned}
m_{14}= & \frac{1}{2}\left(L\left(2 m_{p}+m_{s}\right)-m_{s} r\right) \cos \beta \\
m_{23}= & -\frac{1}{2}\left(L\left(2 m_{p}+m_{s}\right)-m_{s} r\right) \cos \alpha \cos \beta \\
m_{24}= & \frac{1}{2}\left(L\left(2 m_{p}+m_{s}\right)-m_{s} r\right) \sin \alpha \sin \beta \\
m_{33}= & I_{p}+\frac{1}{4}\left(4 I_{s x}+L^{2}\left(4 m_{p}+m_{s}\right)-2 L m_{s} r+m_{s} r^{2}\right) \cos ^{2} \beta+I_{s z} \sin ^{2} \beta \\
m_{44}= & I_{p}+\frac{1}{4}\left(4 I_{s x}+L^{2}\left(4 m_{p}+m_{s}\right)-2 L m_{s} r+m_{s} r^{2}\right) \\
k_{3}= & -\frac{1}{4}\left(4\left(I_{s x}-I_{s z}+L^{2} m_{p}\right)+L^{2} m_{s}-2 L m_{s} r+m_{s} r^{2}\right) \sin (2 \beta) \dot{\alpha} \dot{\beta} \\
& +\frac{1}{2} g\left(L\left(2 m_{p}+m_{s}\right)-m_{s} r\right) \cos \beta \sin \alpha \\
k_{4}= & \frac{1}{8}\left(4\left(I_{s x}-I_{s z}+L^{2} m_{p}\right)+L^{2} m_{s}-2 L m_{s} r+m_{s} r^{2}\right) \sin (2 \beta) \dot{\alpha}^{2} \\
& +\frac{1}{2} g\left(L\left(2 m_{p}+m_{s}\right)-m_{s} r\right) \cos \alpha \sin \beta
\end{aligned}
$$

The rheonomic constraints are the control input $\boldsymbol{u}=\left[\begin{array}{ll}x & y\end{array}\right]^{T} \in \mathbb{R}^{2}$. Note, that in the equation of motion (5) only the accelerations of the carts enter. Linearization around the downward equilibrium point yields two decoupled linearized systems. Applying the Laplace transformation $\mathcal{L}$ these systems are given by

$$
\begin{aligned}
& \alpha(s)=G(s) y(s) \\
& \beta(s)=-G(s) x(s)
\end{aligned}
$$

with

$$
\begin{aligned}
G(s) & =\frac{b_{2} s^{2}}{s^{2}+a_{0}} \\
b_{2} & =\frac{2\left(L\left(2 m_{p}+m_{s}\right)-m_{s} r\right)}{4\left(I_{p}+I_{s x}+L^{2} m_{p}\right)+(L-r)^{2} m_{s}} \\
a_{0} & =g b_{2}
\end{aligned}
$$

where $s \in \mathbb{C}$. Capital letters to denote the Laplace transforms are not used here. It should be emphasized, that in the linear case the $\alpha$ angle is only effected by the displacements in $y$ direction, and the $\beta$ angle by displacements in $x$ direction. Note that for all physically meaningful parameters, $a_{0}$ and $b_{2}$ are positive. In the following the three main parts of the control structure are presented, which enable stabilization, pendular motion in arbitrary planes and tracking of circular motion.

\subsection{Stabilization}

First a stabilizing controller is designed, which should bring the pendulum from arbitrary motion to rest. Therefore, the Laplace transformed dynamical feedback control

$$
y(s)=-V_{\alpha} \underbrace{\frac{s+T_{10}}{s^{3}+T_{22} s^{2}+T_{21} s+T_{20}}}_{K(s)} \alpha(s)
$$




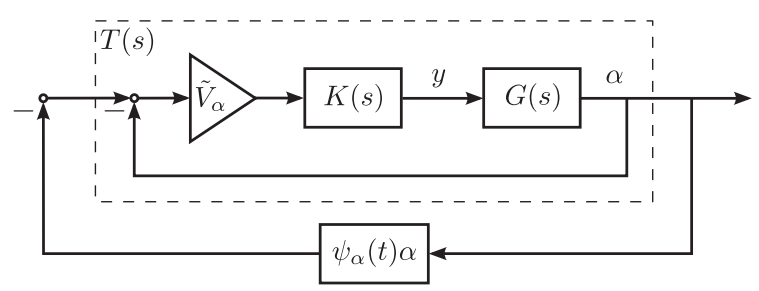

Fig. 4 Control structure for the stabilization

with $T_{10}, T_{22}, T_{21}, T_{20}, V_{\alpha} \in \mathbb{R}$ is employed. Using the Hurwitz criterion it can be shown, that if

$$
T_{10}, T_{21}, T_{20}>0, \quad T_{22}>T_{10}, \quad T_{20}<T_{10} T_{21}, \quad b_{2}, a_{0}>0
$$

hold, the closed loop, i.e. inserting Eq. (10) into Eq. (8), is asymptotically stable for any gain $V>0$. Analogously, we also apply feedback control (10) to the cart's displacement in $X$-direction

$$
x(s)=V_{\beta} K(s) \beta(s)
$$

with any $V_{\beta}>0$. Note, that with the condition given by Eq. (11) the controller $K(s)$ is asymptotically stable and, therefore,

$$
\lim _{t \rightarrow \infty} x(t)=0, \quad \lim _{t \rightarrow \infty} y(t)=0
$$

is directly assured. Thus, the (local) asymptotic stability for $\boldsymbol{q}^{*}=\mathbf{0}$ and $\boldsymbol{u}^{*}=\mathbf{0}$ is achieved.

Independently of the characteristics of $\alpha$ and $\beta$, the inputs $x$ and $y$ are at least one time continuously differentiable $\left(C^{1}\right)$ signals which are also a natural kind of motion of a simple mechanical system, e.g. a driven mass. Additionally, due to the relative degree 2 and the chosen parameters $K(s)$ provides a good noise rejection. Therefore, we can apply a numerical differentiation to get $\dot{x}$ and $\dot{y}$, respectively, and use this signal as a reference for the internal velocity controller.

In order to fulfill the constraints (4) usually $K(s)$ has slow dynamical behavior, e.g. only low amplification in the interesting frequency range, i.e. $V_{\alpha, \beta}$ are chosen small. This causes only very small displacements $x$ and $y$ if $\alpha$ and $\beta$ are not significant. From the root locus method it is known that a small $V$ makes the poles of the closed loop to be very near to the one's of the open loop. Therefore, the resulting closed loop has a poorly damped time response. The closed loop with feedback control (10) and (11) is asymptotically stable for positive $V_{\alpha, \beta}$. Assuming $V_{\alpha}=\tilde{V}_{\alpha}$ as the calculated controller gain according to this feedback, one may suppose that even the increased controller gain $V_{\alpha}=\kappa \tilde{V}_{\alpha}, \kappa>1$ can be applied if the amplitudes of $\alpha$ and $\beta$ are small enough when initializing the controller. In fact, the transient behaviour of the closed loop can be significantly improved by using time-variable controller gains $V_{\alpha, \beta}(t)$. As described in Section 3.2 also the reduced gain $V_{\alpha}=\delta \tilde{V}_{\alpha}, \delta \in(0,1)$ is of interest. In summary, the stability of the closed loop as shown in Figure 4 should be analyzed, where $\psi_{\alpha}$ is a bounded, piecewise continuous function with

$$
-1+\delta \leq \psi_{\alpha}(t) \leq \kappa-1 .
$$

From a practical point of view fully sufficient conditions are given by $\delta=0.1$ and $\kappa=2$. The (robust) asymptotic stability of this closed loop can be proven by using the circle-criterion $[10,11]$, where $T(s)$ is calculated by setting $\tilde{V}_{\alpha}=0.85, T_{10}=1.184, T_{20}=2.979$, $T_{21}=2.951, T_{22}=2.673$. The same result holds for the stabilization of $\beta$.

The controller gain $V_{\alpha, \beta}(t)=\tilde{V}_{\alpha, \beta}\left(1+\psi_{\alpha, \beta}(t)\right)$ is designed as follows. Let ${ }_{i} A_{\alpha}$ be the maximum of the absolute values of $\alpha(t)$, where $t \in\left[(i-1) T_{A}, i T_{A}\right), i \in \mathbb{N}$ with the interval length $T_{A}>0$, i.e.

$$
{ }_{i} A_{\alpha}=\max _{(i-1) T_{A} \leq t<i T_{A}}|\alpha(t)|, \quad{ }_{0} A_{\alpha}=\alpha_{\max } .
$$


Assembling these ${ }_{i} A_{\alpha}$ to the function $A_{\alpha}(t)$ leads to

$$
\begin{aligned}
h(t) & = \begin{cases}1 & \text { if } \quad 0 \leq t<T_{A}, \\
0 & \text { else },\end{cases} \\
A_{\alpha}(t) & =\sum_{i \geq 1}{ }_{i} A_{\alpha} h\left(t-i T_{A}\right)+{ }_{0} A_{\alpha} h(t) .
\end{aligned}
$$

The controller gain $V_{\alpha}(t)$ is now chosen to be

$$
V_{\alpha}(t)=V_{\alpha}\left(A_{\alpha}(t)\right), \quad \delta \tilde{V}_{\alpha} \leq V_{\alpha}(t) \leq \kappa \tilde{V}_{\alpha} .
$$

The same result holds for $V_{\beta}(t)$. With $A_{\alpha, \beta}$ being piecewise constant, functions $V_{\alpha, \beta}$ are also piecewise constant. Therefore, the applied closed loop contains a switching controller. It should be pointed out that $\psi(t)$ is a simplified notation since $\psi$ also depends on values of $y$. Thus, the function $\psi$ cannot be called a memoryless function which is used in the proof of the circle-criterion. Due to the special form of $\psi$ the circle-criterion is still applicable. Note that a switching of $V_{\alpha, \beta}$ does not affect the $C^{1}$ property of the input signals $x$ and $y$.

Figure 5 shows the curves of $\alpha, y$ and the controller gain $V_{\alpha}$ when stabilizing the system. In the simulation the value $T_{A}$ in Eq. (15) is set to $2.9 \mathrm{~s}$ and the initial conditions are $\boldsymbol{q}(0)=$ $\left(30^{\circ}, 0\right)^{T}$ and $\dot{\boldsymbol{q}}(0)=(0,0)^{T}$. Clearly, the plots show that the time response of the closed loop with $V_{\alpha}=\tilde{V}=$ constant is less damped than the response with modified controller gain. For both controllers the position constraint as well as the constraints on the velocity and acceleration are fulfilled, see Fig. 6.
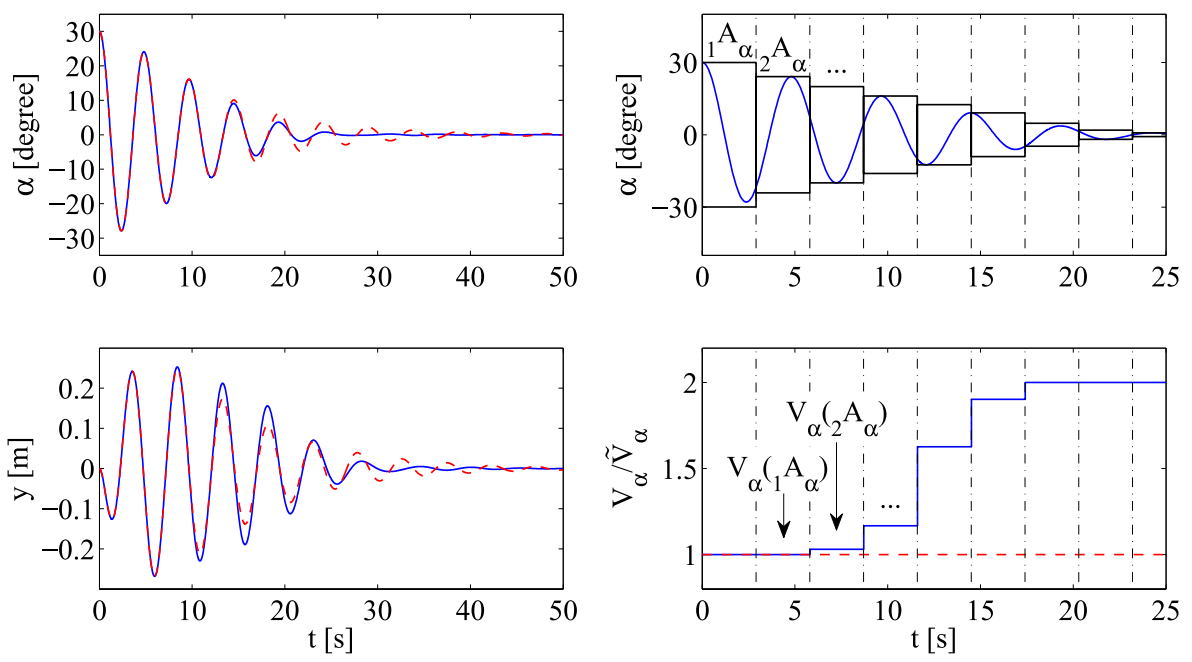

Fig. 5 Stabilizing by linear controller with constant (dashed) and piecewise constant (solid) controller gains.
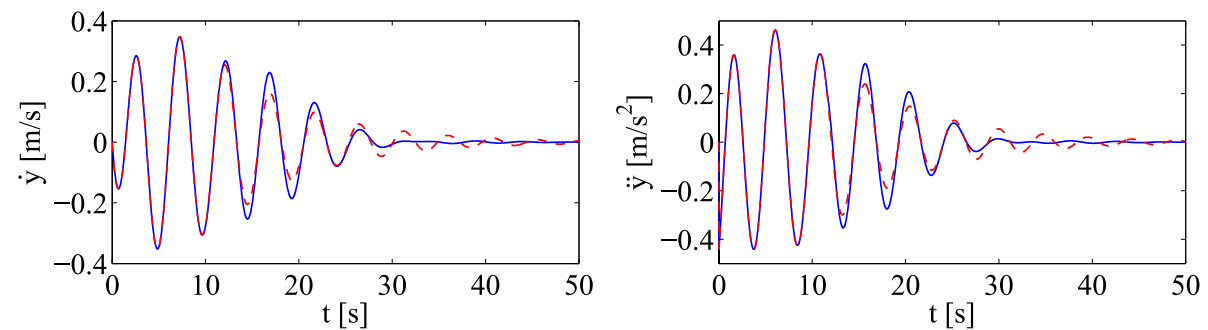

Fig. 6 Checking the velocity and acceleration constraints while stabilizing by the controllers as in Fig. 5. 


\subsection{Pendular motion in arbitrary plane}

The previously designed stabilizing control can be extended to bring the pendulum from an arbitrary motion into a pendular motion of the sphere in a desired $X Z$-plane rotated along the $Z$-axis. Therefore, the measured $\alpha$ and $\beta$ are transformed into $\bar{\alpha}$ and $\bar{\beta}$ which describe the sphere's position in the new coordinate system $\bar{X} \bar{Y} \bar{Z}$ that is derived from the $X Y Z$-coordinate system by rotating it along the $Z$-axis with the angle $\gamma$. Making $\bar{\alpha}$ to zero is equivalent to bringing the sphere's center point into a pendular motion in the $\bar{X} \bar{Z}$-plane. The transformed Cardan angles $\bar{\alpha}$ and $\bar{\beta}$ can be calculated as

$$
\begin{aligned}
& \bar{\beta}=\bar{\beta}(\alpha, \beta, \gamma)=\arcsin (\sin \beta \cos \gamma-\sin \alpha \cos \beta \sin \gamma), \\
& \bar{\alpha}=\arcsin \left(\frac{\sin \beta \sin \gamma+\sin \alpha \cos \beta \cos \gamma}{\cos \bar{\beta}(\alpha, \beta, \gamma)}\right),
\end{aligned}
$$

where $|\bar{\alpha}|,|\bar{\beta}|<\frac{\pi}{2}$. Equivalently to Eq. (8) it can be directly stated that the dynamical behavior of the system is

$$
\bar{\alpha}(s)=G(s) \bar{y}(s), \quad \bar{\beta}(s)=-G(s) \bar{x}(s)
$$

where

$$
\left[\begin{array}{l}
\bar{x} \\
\bar{y}
\end{array}\right]=\underbrace{\left(\begin{array}{rr}
\cos \gamma & \sin \gamma \\
-\sin \gamma & \cos \gamma
\end{array}\right)}_{\boldsymbol{C}}\left[\begin{array}{l}
x \\
y
\end{array}\right] .
$$

Thus, in the linear case the motion in two perpendicular planes can be stabilized independently. For large $\alpha, \beta$ there exists a slight coupling of the motions in the perpendicular planes. The resulting control structure is shown in Figure 7. This can be used for example to rotate the pendular plane or to switch from circular motion into a pendular motion. In this case only light damping is injected in the desired direction of the pendular motion, while strong damping is injected in the perpendicular direction. Thus, finally only pendular motion in the desired plane remains.

The excitation of pendular motion from rest or the increasing of the amplitude is achieved by an open loop control. For pendular motion in one of the planes parallel to the crosstable axis, only this axis is excited. Thereby the cart performs a sinusoidal motion, and the pendulum is driven close to resonance until the desired amplitude is achieved. In the meanwhile, the stabilization control is applied to the other axis which is perpendicular to the pendular motion. The excitation amplitude of the cart is decreased when approaching the desired amplitude using a dynamic lock-up table. As soon as the desired amplitude is reached, also the stabilization control for this axis is activated, where an amplitude dependent control gain is used for noise rejection. If the amplitude decreases due to damping after some time, the system is

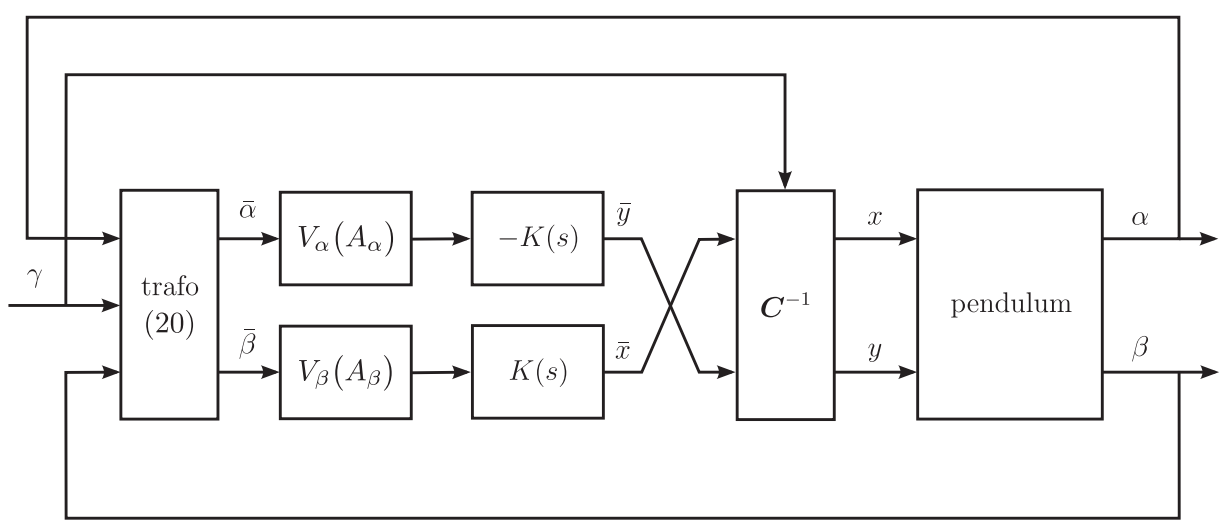

Fig. 7 Control scheme for independent stabilization in two orthogonal planes 
again excited. In order to excite pendular motion in an arbitrary rotated plane, the previously presented rotation and decomposition structure is used. Then, pendular motion in an arbitrary rotated plane is again achieved by open-loop control, while the perpendicular axis is stabilized by strong damping.

\subsection{Tracking of circular motion}

Finally a tracking controller should be designed such that the sphere's center point $\left(w_{x}, w_{y}\right)$ from Eq. (3) asymptotically tracks circles in the $X Y$-plane. Therefore, the transfer function from $x$ and $y$ to $w_{x}$ and $w_{y}$, respectively, are derived. With the approximation

$$
\beta \approx \frac{w_{x}-x}{L}, \quad \alpha \approx-\frac{w_{y}-y}{L},
$$

we obtain from Eq. (8)

$$
\begin{aligned}
& w_{x}(s)=\overbrace{G_{w}(s)}^{\frac{\overbrace{\left(1-b_{2} L\right)}^{\tilde{b}_{2}} s^{2}+a_{0}}{s^{2}+a_{0}}} x(s), \\
& w_{y}(s)=G_{w}(s) y(s) .
\end{aligned}
$$

The desired circular trajectories in the $X Y$ plane yield sinusoids trajectories with a frequency $\omega_{0}$ in the $x$ and $y$ direction. Therefore, the internal model principle is used to design a tracking controller for Eqs. (22) and (23). Thus, we set the tracking controller $K_{T}(s)$ in the closed loop as shown in Figure 8 as

$$
K_{T}(s)=\frac{\sum_{i=0}^{m} c_{i} s^{i}}{\sum_{i=0}^{m} d_{i} s^{i}} \frac{1}{s^{2}+\omega_{0}^{2}} \frac{\frac{s}{T_{2}}+1}{\frac{s}{T_{1}}+1}, \quad c_{i}, d_{i} \in \mathbb{R}, d_{m}=1, m \in \mathbb{N}, T_{1}, T_{2} \in \mathbb{R}, T_{1}>0 .
$$

The first term is used for stabilization, the second term follows from the internal model principle and the third term yields offset and noise rejection. Due to the similarity of Eqs. (22) and (23) we only deal with Eq. (22). The error dynamics is given by

$$
e_{x}(s)=\mathcal{L}\left\{w_{x}^{*}-w_{x}\right\}=\frac{1}{1+G_{w}(s) K_{T}(s)} \frac{R}{s^{2}+\omega_{0}^{2}}
$$

which gives us the characteristic equation for the tracking error as

$$
\left(\frac{s}{T_{1}}+1\right)\left(s^{2}+\omega_{0}^{2}\right)\left(s^{2}+a_{0}\right)\left(\sum_{i=0}^{m} d_{i} s^{i}\right)+\left(\tilde{b}_{2} s^{2}+a_{0}\right)\left(\sum_{i=0}^{m} c_{i} s^{i}\right)\left(\frac{s}{T_{2}}+1\right)=0 .
$$

The first part of Eq. (26) is a polynomial of degree $m+5$ and, therefore, we have to design $m+5$ coefficients in order to place the $m+5$ poles of Eq. (26). On the other hand there are $2 m+1$ unknowns from Eq. (24) which leads to

$$
2 m+1 \stackrel{!}{=} m+5 \quad \longleftrightarrow \quad m=4
$$

Let the desired characteristic equation be

$$
s^{9}+p_{8} s^{8}+\ldots+p_{1} s+p_{0}=0, \quad p_{i} \in \mathbb{R} .
$$

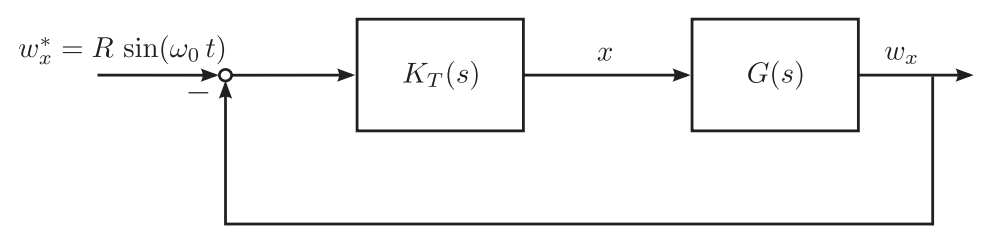

Fig. 8 Linear tracking control for $w_{x}$ 
Comparing the coefficients of Eqs. (26) and (28) gives the linear equation

$$
\boldsymbol{A} \boldsymbol{n}=\boldsymbol{b}
$$

with

$$
\boldsymbol{n}=\left[\begin{array}{lllllllll}
c_{4} & c_{3} & c_{2} & c_{1} & c_{0} & d_{3} & d_{2} & d_{1} & d_{0}
\end{array}\right]^{T}, \quad \boldsymbol{n} \in \mathbb{R}^{9}
$$

and a certain matrix $\boldsymbol{A} \in \mathbb{R}^{9 \times 9}$ and vector $\boldsymbol{b} \in \mathbb{R}^{9}$.

Note that since both $K(s)$ from Eq. (10) and $K_{T}(s)$ from Eq. (24) have relative degree 2, the input signal for the cart position control is $C^{1}$ even while switching between these kind of controllers. The frequency $\omega_{0}$ should be chosen properly to ensure the constraints (4) to be fulfilled. In order to achieve small motions of the carts, the choice of the frequency $\omega_{0}$ is oriented towards the circular motion with radius $R$ of a pendulum with fixed carts. This, can be, e.g., determined using a optimization procedure, for details see [9].

\section{Experimental Results}

This section provides some experimental results of the real-life EXPO-pendulum, which were performed on the test rig in Stuttgart, presented in Fig. 1. In the first experiment the free uncontrolled pendulum motion is analyzed. Figure 9 shows the angle $\beta$ which is subjected to an initial disturbance. The plot shows clearly the natural frequency of approximately $0.21 \mathrm{~Hz}$ and the only very small damping of the free uncontrolled pendulum. The damping results from small friction in the Cardan joint and, in addition, for larger angles from wind resistance.

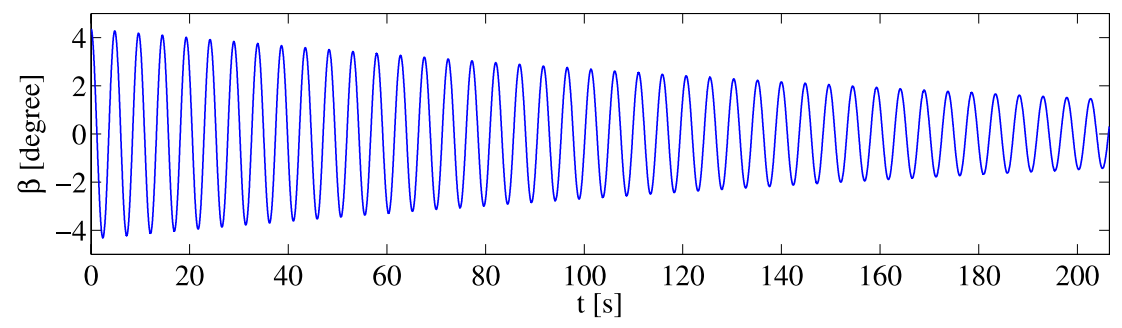

Fig. 9 Experiment 1: Free motion of the pendulum.

In the second experiment the sphere is accelerated from rest into a circular motion, then the motion is changed into a pendular motion and finally the pendulum is brought back to rest. Figure 10 shows the trajectory of the sphere's center of mass and in Fig. 11 the corresponding Cardan angles $\alpha, \beta$ and the control input, i.e. the cart displacements, $x, y$ are given. First the sphere is accelerated from rest up to an circular motion with $2.3 \mathrm{~m}$ radius, using the tracking controller presented in Section 3.3. After only $40 s$ the desired radius is reached and this radius is hold. For the start-up phase the trajectory of the sphere's center of mass and the cross table's cart are compared in Fig. 12. This shows clearly the huge difference between the cart's small motion and the large sphere motion. Thus the motion remains close to the circular motion of a free pendulum which is desired to create an impressive show. Figure 13 shows for this phase the desired and measured position of the center of mass of the sphere, and the resulting trajectory error. It is seen that the tracking controller yields very good tracking of the desired circular motion in the entire phase. Most of time the position error is less than $3 \mathrm{~cm}$.

In the second phase of this experiment the pendulum motion changes from circular motion into a planar pendular motion in the $X Z$-plane, i.e. only motion of the $\beta$ angle. For this the controller presented in Section 3.2 is used. This transition starts at time $60 \mathrm{~s}$. First the motion in the perpendicular direction is damped out, as it can be clearly seen in the plot of the angle $\alpha$, presented in Fig. 11. This transition is also observed in the control action of the cart in $y$ direction, which influences the $\alpha$ angle. Note, that for large angles, both Cardan 


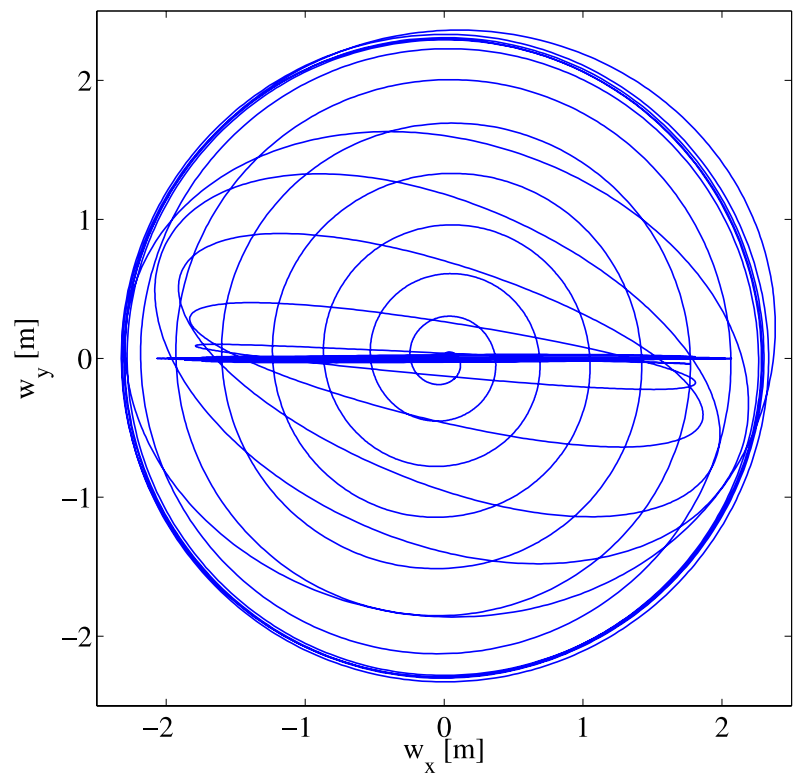

Fig. 10 Experiment 2: Trajectory of the sphere's center of mass.
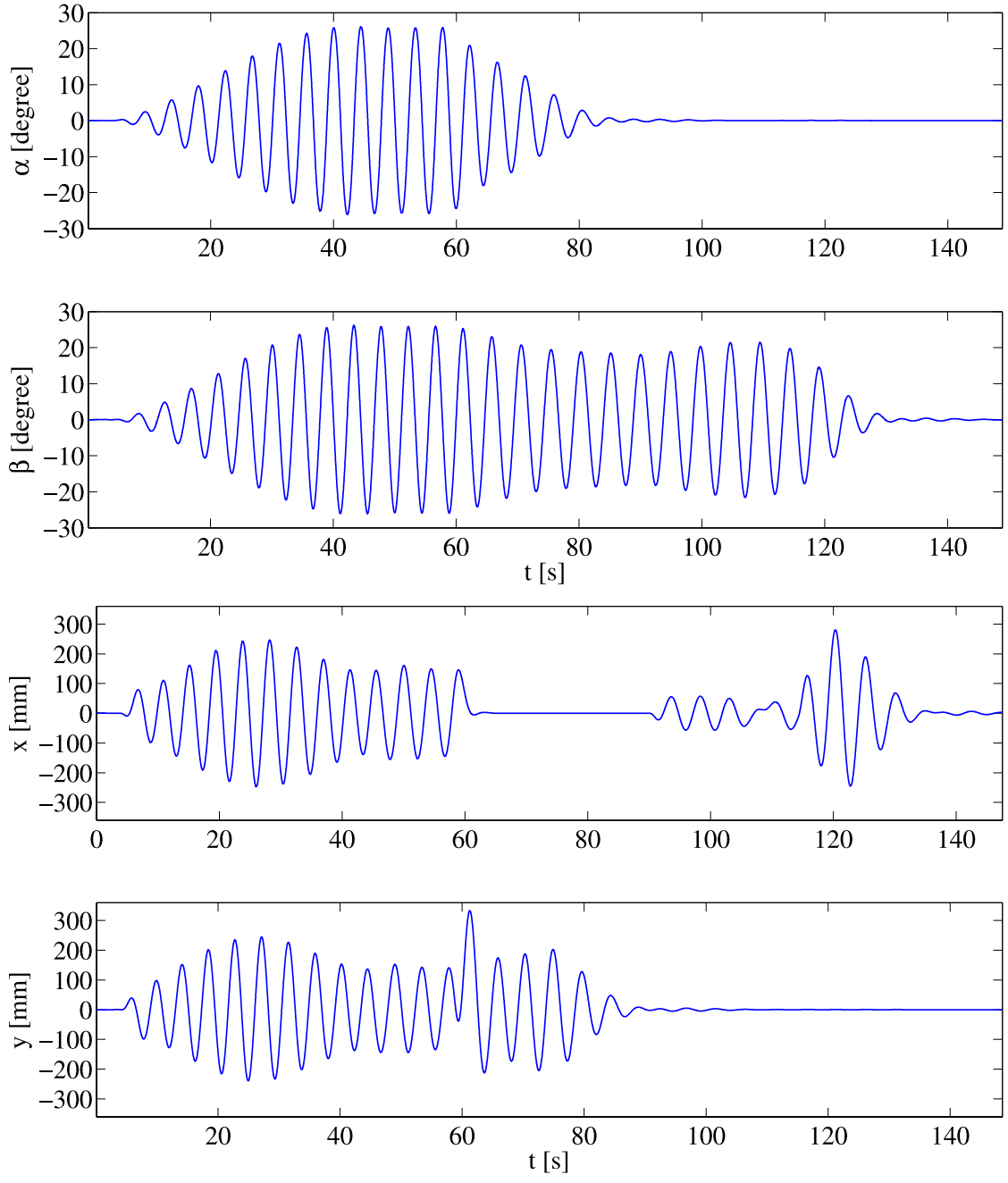

Fig. 11 Experiment 2: Cardan angles and displacements of the carts. 
angles are increasingly coupled by the nonlinear equation of motion (1). This yields that in the transition phase a rotated ellipsoidal trajectory occurs and the $\beta$ angle is slightly reduced. After approximately $25 s$ no significant motion in the perpendicular plane is left. Now the final desired amplitude in the $X Z$-plane is increased until the desired value is reached. This can be seen from the little control action of the cart in $x$ direction.

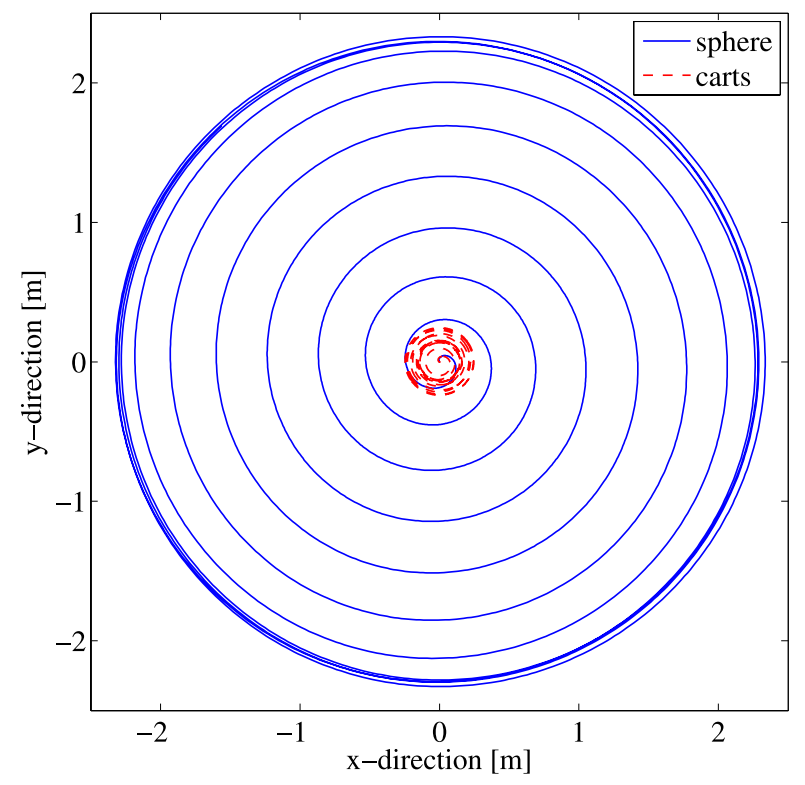

Fig. 12 Experiment 2: Sphere and cart trajectories during circular motion.
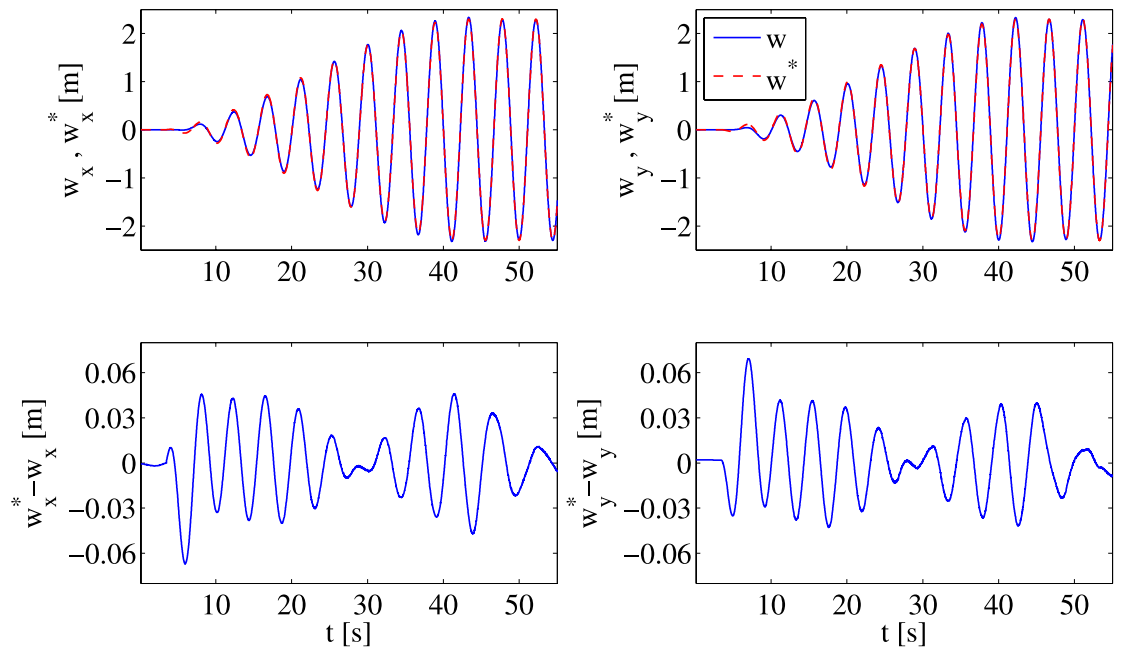

Fig. 13 Experiment 2: Position and position error of the sphere during circular motion.

In the last part of the second experiment, the pendulum motion is damped out and the pendulum is brought back to rest. This breaking starts at approximately $110 \mathrm{~s}$. As it can be seen from the displacement of the cart in $x$ direction and the $\beta$ angle, no significant motion is left after $20 s$, see Fig. 11. This fast breaking shows the efficiency of the stabilizing control law derived in Section 3.1.

In a third experiment the pendulum is first brought into pendular motion in the rotated $\bar{X} \bar{Z}$-plane, described by $\gamma=22.5^{\circ}$. Then, the plane is rotated into the $X Z$-plane. For this experiment Fig. 14 contains the trajectory of the sphere's center of mass and in Fig. 15 the corresponding Cardan angles $\alpha, \beta$ and the control input, i.e. the cart displacements $x, y$. The 


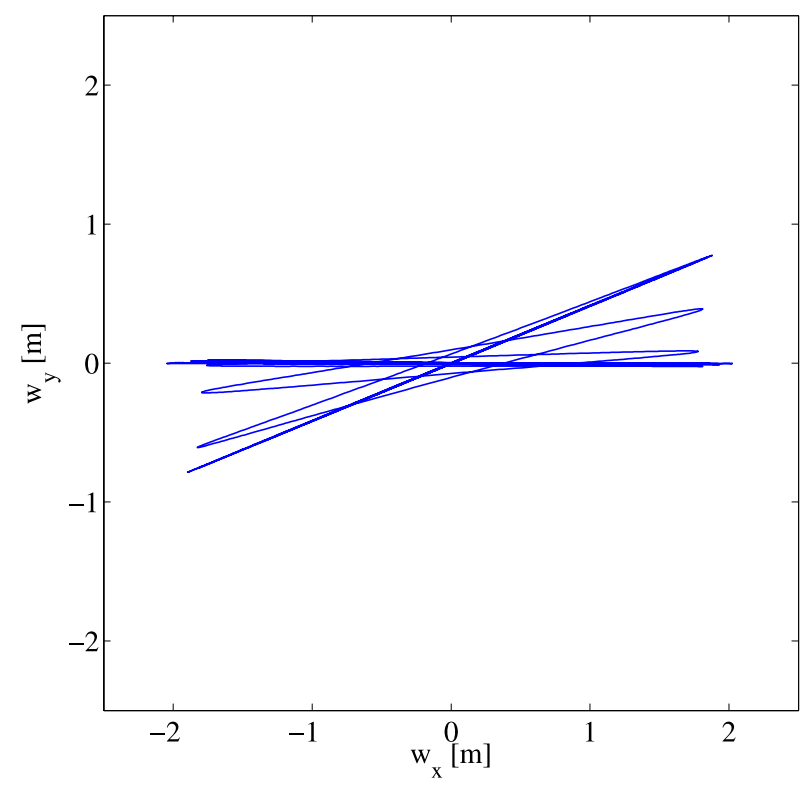

Fig. 14 Experiment 3: Trajectory of the sphere's center of mass.
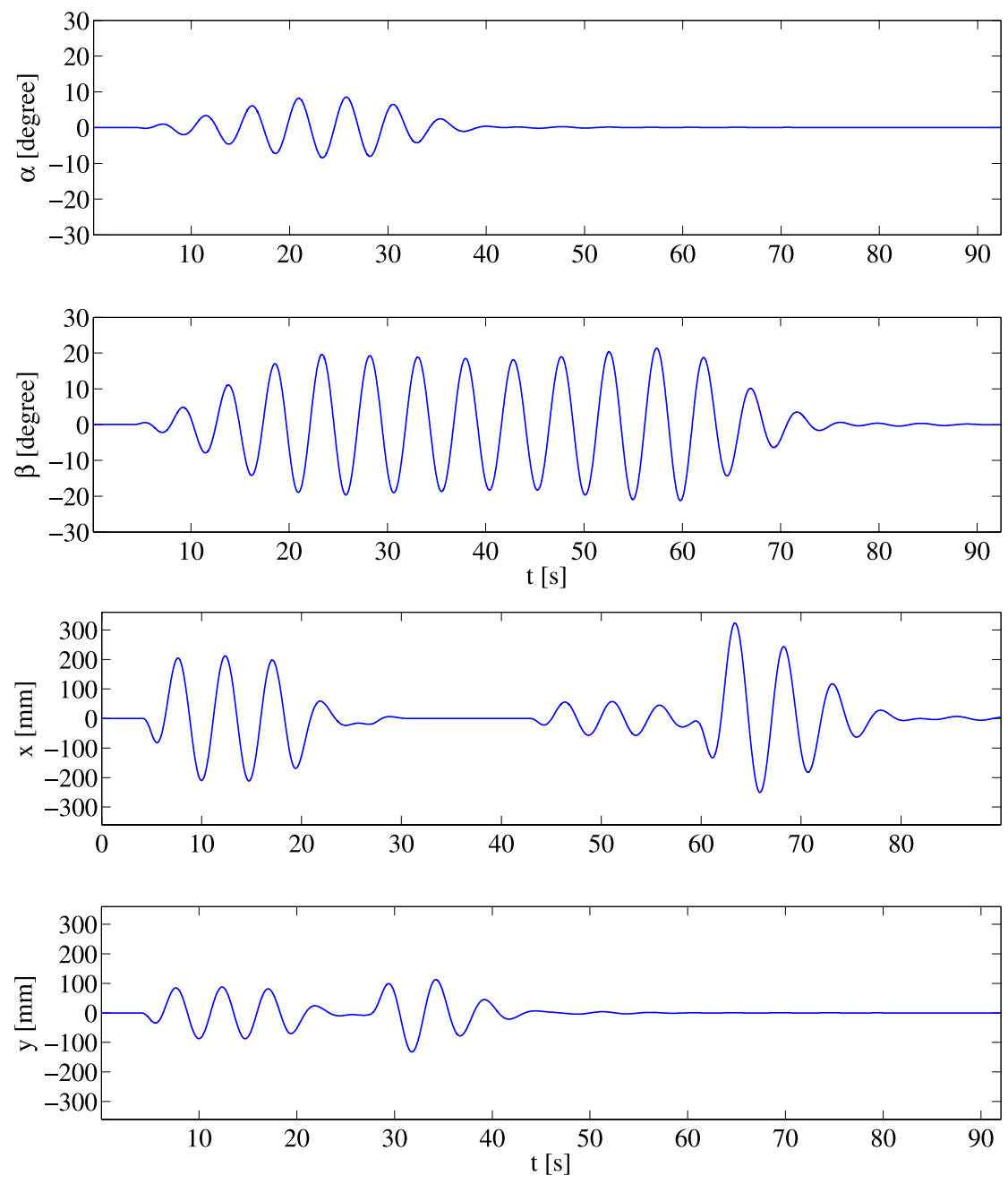

Fig. 15 Experiment 3: Cardan angles and displacements of the carts. 
start-up phase is approximately $25 \mathrm{~s}$ long. Then the desired amplitude of $2.1 \mathrm{~m}$ of the pendular motion in the $\bar{X} \bar{Z}$-plane is reached. In this case both carts are producing control inputs, exciting both angles. Thereby, due to the rotation of $\gamma=22.5^{\circ}$ the excitation of the $\beta$ angle is much stronger. The combined motion yields the pendular motion in the rotated $\bar{X} \bar{Z}$-plane.

In the second phase of this experiment the pendular plane is rotated into the $X Z$-plane, which starts at time $25 \mathrm{~s}$. For this the controller from Section 3.2 is used with the rotation angle $\gamma=0^{\circ}$. Now, all motion perpendicular to the $X Z$-plane is damped out by the stabilization controller, while in the $X Z$-plane the pendular motion remains. During this transition only the cart in $y$-direction produces control input which yields the stabilization of the $\alpha$ angle. After $15 s$ all significant motion in the perpendicular plane is damped out. Due to the rotation, the amplitude of the pendular motion in the $X Z$-plane decreases. After rotation of the plane, the amplitude is increased again. In this case only significant control action of the cart in $x$ direction occurs, yielding only motion of the $\beta$ angle. However, note that in this time the stabilization control is active for the perpendicular plane, in order to compensate for disturbances in the $\alpha$ angle. In the third part of the last experiment, the pendulum motion is again brought to rest. Similar to the previous experiment this is achieved in about $20 \mathrm{~s}$.

\section{Conclusion}

The large interactive 3D-show-pendulum for the German Pavilion at the World Exhibition EXPO 2010 in Shanghai was presented. For the desired shows, stabilization of the equilibrium point as well as pendular motion and trajectory tracking of circular motion must be achieved. Therefore, a modified linear control approach was developed and implemented on the real-life system. This approach is very well suitable to meet the control goals and the technical limitations of the actual hardware. The efficiency of this approach was shown by experiments on the real-life system.

\section{Acknowledgements}

Such a large project is the work of many people. Especially the authors want to thank Milla \& Partners and Mr. Ingo Kaske who are responsible for the idea, concept and the coordination, the Institute for Control Engineering of Machine Tools and Manufacturing Units (ISW) (Dipl.-Ing. A. Hafla, Dipl.-Ing. H.-P. Bock, Dipl.-Ing. P. Sekler) who worked on the drives, sensors and control hardware, and the Institute of Machine Components (IMA) (Dr.Ing. A. Krolo, Dipl.-Ing. B. Klein) who did the initial design of the mechanical components. The actual manufacturing was done by Metron and the video hardware is from ICT. The German Pavilion has been commissioned by the Federal Ministry of Economics and Technology, organization and operation was conducted by Koelnmesse International GmbH. 


\section{References}

( 1 ) Singhal, R., Patayane, R. and Banavar R.N., Tracking a Trajectory for a Gantry Crane: Comparision Between IDA-PBC and Direct Lyapunov Approach, IEEE International Conference on Industrial Technology, pp. 1788-1793, 2006.

( 2 ) Wai, R.-J. and Chang, L.-J., Adaptive Stabilizing and Tracking Control for a Nonlinear Inverted-Pendulum System via Sliding-Mode Technique, IEEE Transactions on Industrial Electronics, 53(2), pp. 674-692, 2006.

( 3 ) Fang, Y., Dixon, W.E., Dawson, D.M. and Zergeroglu, E., Nonlinear Coupling Control Laws for a 3-DOF Overhead Crane System, Proceedings of the 40th IEEE Conference on Decision and Control, 4, pp. 3766-3771, 2001.

( 4 ) Martindale, S.C., Dawson, D.M., Zhu, J. and Rahn, C.D., Approximate Nonlinear Control for a Two Degree of Freedom Overhead Crane: Theory and Experimentation, Proceedings of the American Control Conference, 1, pp. 301-305, 1995.

( 5 ) Ortega, R., Spong, M.W. , Gómez-Estern, F. and Blankenstein, G., Stabilization of a Class of Underactuated Mechanical Systems Via Interconnection and Damping Assignment, IEEE Transactions on Automatic Control, 47(8), pp. 1218-1233, 2002.

( 6 ) Aschemann, H., Passivity-Based Trajectory Control of an Overhead Crane by Interconnection and Damping Assignment, In Motion and Vibration Control: Selected Papers from MOVIC 2008, H. Ulbrich, L. Ginzinger (Eds.), Springer, Dordrecht, pp. 21-30, 2008.

( 7 ) http://www.itm.uni-stuttgart.de/research/expo2010/expo2010_en.php

( 8 ) Kurz, T., Eberhard, P., Henninger, C. and Schiehlen, W., From Neweul to Neweul-M²: Symbolical Equations of Motion for Multibody System Analysis and Synthesis, Multibody System Dynamics, 24(1), pp. 25-41, 2010.

( 9 ) Gorius, T., Seifried, R., Eberhard, P., Control Approaches for a 3D-Pendulum on Display at the EXPO 2010. In Proceedings of the International Symposium on Coupled Methods in Numerical Dynamics, Z. Terze, C. Lacor (Eds.), Faculty of Mechanical Engineering and Naval Architecture, Zagreb, pp. 281-300, 2009.

(10) Vidyasagar, M.: Nonlinear Systems Analysis. 2. Edition, Prentice Hall, Englewood Cliffs, New Jersey, 1993.

(11) Gorius, T., Seifried, R., Eberhard, P., Regelung des EXPO-Pendels (in German). atAutomatisierungstechnik, 59, pp. 299-307, 2011. 